CLINICAL STUDY

\title{
Phase II study of weekly paclitaxel and sorafenib as second/third-line therapy in patients with adrenocortical carcinoma
}

\author{
Alfredo Berruti, Paola Sperone, Anna Ferrero, Antonina Germano ${ }^{1}$, Arianna Ardito ${ }^{1}$, Adriano \\ Massimiliano Priola ${ }^{2}$, Silvia De Francia ${ }^{3}$, Marco Volante ${ }^{4}$, Fulvia Daffara ${ }^{1}$, Daniele Generali ${ }^{5}$, Sophie Leboulleux ${ }^{6}$, \\ Paola Perotti, Eric Baudin ${ }^{6}$, Mauro Papotti ${ }^{4}$ and Massimo Terzolo ${ }^{1}$ \\ Medical Oncology, ${ }^{1}$ Internal Medicine, ${ }^{2}$ Radiology, ${ }^{3}$ Pharmacology and ${ }^{4}$ Pathology, Department of Clinical and Biological Science, University of Turin, \\ Azienda Ospedaliero Universitaria San Luigi, Regione Gonzole 10, 10043 Orbassano, Italy, ${ }^{5}$ Breast Cancer Molecular Unit, Azienda Ospedaliera Istituti \\ Ospitalieri Cremona, Cremona, Italy and ${ }^{6}$ Service de Médecine Nucléaire et de Cancérologie Endocrinienne, Institut Gustave-Roussy, Université Paris XI, \\ Villejuif, France \\ (Correspondence should be addressed to A Berruti; Email: alfredo.berruti@gmail.com)
}

\begin{abstract}
Background: There is a strong rationale in the use of antiangiogenic therapy in the management of adrenocortical carcinoma (ACC). Metronomic administration of chemotherapy and antiangiogenic drugs can be synergistic in targeting endothelial cells.

Objective: We assessed the activity of sorafenib plus metronomic paclitaxel as second/third-line therapy in advanced ACC patients. We also tested the activity of sorafenib and paclitaxel against NCI-H295R in vitro.

Design: Multicenter, prospective phase II trial.

Setting: Referral centers for ACC.

Methods: Twenty-five consecutive metastatic ACC patients who progressed after mitotane plus one or two chemotherapy lines were planned to be enrolled. The patients received a combination of i.v. paclitaxel $\left(60 \mathrm{mg} / \mathrm{m}^{2}\right.$ every week) and oral sorafenib (400 $\mathrm{mg}$ twice a day) till progression. The primary aim was to measure the progression-free survival rate after 4 months and the secondary aims were to assess the objective response rate and toxicity.

Results: Tumor progression was observed in nine evaluable patients at the first assessment. These results led to the premature interruption of the trial. The treatment was well tolerated. The most relevant toxicities were fatigue, being grade 2 or 3 in four patients, and hypophosphatemia, being grade 3 in three patients. In the in vitro study, sorafenib impaired the viability of H295R cells with dose-response and time-response relationships. The in vitro sorafenib activity was not increased in combination with paclitaxel.

Conclusions: Despite the in vitro activity, sorafenib plus weekly paclitaxel is an inactive salvage treatment in patients with advanced ACC and should not be recommended.

European Journal of Endocrinology 166 451-458
\end{abstract}

\section{Introduction}

Adrenocortical carcinoma (ACC) is a highly malignant disease that is resistant to medical treatment. Surgery is the only treatment potentially able to cure ACC patients (1), but when ACC cannot be surgically removed, or the tumor recurs after surgery, prognosis is dismal $(1,2,3)$.

The efficacy of the currently used chemotherapy regimens is modest at best, and most patients are destined to relapse. Therefore, there is an urgent need for new treatment strategies (4).

Metronomic chemotherapy is the administration of antineoplastic drugs at low doses, on a frequent or continuous schedule, with no extended interruptions (5). This treatment modality targets tumor cells indirectly via inhibiting angiogenesis and vasculogenesis by continuously exposing the more slowly proliferating tumor endothelial cells to cytotoxic therapy $(5,6)$. Lowdose metronomic chemotherapy may offer several advantages, including low toxicity and treatment response irrespective of the resistance profile of the tumor cell population $(5,7,8)$.

Paclitaxel is an anticancer agent that has been found to be effective in the treatment of several malignancies (9). It promotes polymerization of tubulin dimers to form microtubules and stabilizes microtubules by preventing depolymerization (9). Preclinical studies have shown that paclitaxel is active against ACC cell lines (10). Paclitaxel administered on a weekly metronomic schedule was found to be more effective than the 
3-week administration in two randomized prospective clinical trials involving breast cancer patients either as a neoadjuvant approach before primary surgery (11) or in advanced disease (12). Paclitaxel, therefore, seemed to be a good candidate to be tested in a metronomic schedule in advanced ACC patients.

Since ACC is highly vascularized (13) and shows high levels of vascular endothelial growth factor (VEGF) (14), we believed that the introduction of a small molecule targeting the angiogenesis pathways at different levels could be a good strategy to be used in ACC treatment.

Sorafenib is an inhibitor of several receptor tyrosine kinases involved in neovascularization, including VEGFR2, VEGFR3, and platelet-derived growth factor. Sorafenib also inhibits RAF-1, a key enzyme in the RAS/RAF/MEK/ERK signaling pathway leading to cell proliferation (15). Sorafenib was approved by the FDA for the treatment of advanced renal cell cancer and hepatocellular cancer and shows good clinical activity in thyroid cancer (16). Sorafenib showed a broad spectrum of antitumor activity in preclinical studies (15), so multiple clinical trials are undertaken to further investigate the role of sorafenib alone or in combination with paclitaxel for the treatment of various tumor entities.

As suggested by Kerbel \& Kamen (5), there is a strong rationale for combining metronomic chemotherapy with antiangiogenic drugs. Before preclinical metronomic chemotherapy, studies have shown that the combination of antiangiogenic drugs with metronomic chemotherapy can increase the antitumor efficacy compared with either agent alone $(17,18)$. Two nonrandomized phase II trials have tested the association of metronomic chemotherapy plus bevacizumab in advanced ovarian and breast cancers, respectively, and promising results have been obtained $(19,20)$.

On these bases, a prospective, open-label, single-arm, multicenter study was planned with the aim to determine the activity and toxicity of sorafenib plus weekly paclitaxel as a second/third-line approach in patients with advanced/metastatic ACC.

The objective of the primary study was to assess the clinical benefit as measured by the nonprogressing rate after 4 months of the combination regimen. The secondary aims were to assess the objective response rates and toxicity. In order to obtain information on the interaction between the two drugs, an in vitro study was also performed to test the antiproliferative effect of either drug, alone or in combination, against NCI-H295R ACC cells.

\section{Materials and methods}

\section{Patients and methods}

To be included in this trial, the patients had to meet the following inclusion criteria: age $\geq 18$ years, histologically confirmed diagnosis of ACC, locally advanced or metastatic disease not amenable to surgery, progressive disease after one or two cytotoxic chemotherapy regimens (including a cisplatin-based protocol), Eastern Cooperative Oncology Group (ECOG) performance status $0-2$, life expectancy $\geq 3$ months, and at least one unidimensional (RECIST criteria) measurable lesion, adequate bone marrow reserve (neutrophils $\geq 1500 / \mathrm{mm}^{3}$ and platelets $\geq 100000 / \mathrm{mm}^{3}$ ), hemoglobin $\geq 9.0 \mathrm{~g} / \mathrm{dl}$, total bilirubin $\leq 1.5$ times the upper limit of normal, PT-INR/PTT $<1.5 \times$ upper limit of normal, serum creatinine $\leq 1.5 \times$ upper limit of normal, effective contraception in premenopausal female and male patients, written informed consent, and ability to comply with the protocol procedures.

Exclusion criteria were history of prior malignancy, except for cured nonmelanoma skin cancer, cured in situ cervical carcinoma, or other treated malignancies with no evidence of disease for at least 3 years, active clinically serious infections (greater than grade 2 National Cancer Institute - Common Toxicity Criteria (NCI-CTC) version 3.0), symptomatic metastatic brain or meningeal tumors, seizure disorder requiring medication (i.e. steroids or antiepileptics), concomitant rifampicin, concomitant St. John's Wort (Hypericum perforatum), decompensated heart failure (ejection fraction $<45 \%$ ), myocardial infarction or revascularization procedure during the last 6 months, unstable angina pectoris, uncontrolled cardiac arrhythmia, hypertension that was not controlled by medications ( $>160 / 100 \mathrm{mmHg}$ despite optimal medical therapy), patients with recent or active bleeding diathesis, pregnant or breast-feeding patients, previous treatment with sorafenib, other anticancer chemotherapy or immunotherapy during the study or within 4 weeks of study entry, radiotherapy during study or within 3 weeks of study start (palliative radiotherapy was allowed), major surgery within 4 weeks of start of study, current treatment with another investigational drug, any other severe acute or chronic medical or psychiatric condition, or laboratory abnormality which, in the judgment of the investigator, would have made the patient inappropriate for entry into this study. The investigation was approved by the Local Ethics Committee and was conducted according to Good Clinical Practice. Written consent was obtained from each patient after full explanation of purpose and nature of all procedures used.

Treatment schedule Sorafenib was administered orally at the dose of $400 \mathrm{mg}$ twice a day and paclitaxel was administered i.v. at the dose of $60 \mathrm{mg} / \mathrm{m}^{2}$ every week. The treatment was planned to be continued until disease progression. Mitotane was withdrawn 1 month before sorafenib initiation till the end of the study.

In order to prevent major hypersensitivity reactions, a premedication with corticosteroids (dexamethasone, $4 \mathrm{mg}$ i.v.), H1-receptor antagonist (diphenhydramine, $50 \mathrm{mg}$ i.v.), and H2-receptor antagonists (cimetidine, $300 \mathrm{mg}$ or ranitidine, $150 \mathrm{mg}$ i.v.) was given before paclitaxel administration. 
Recommended treatment modifications for hematological toxicities were as follows: if granulocyte counts were $<1.5 \times 10^{9} / \mathrm{l}$ and/or platelet counts were $<100 \times 10^{9} / 11$ week, a delay of paclitaxel was introduced while sorafenib was decreased by one dose level. In case of lower hematological values, paclitaxel was delayed until resolution and sorafenib delayed until recovery of at least grade 2 toxicity. Then, sorafenib was reduced by one dose and paclitaxel administered at $60 \%$ of dose.

As far as nonhematological toxicities were concerned, in case of grade 4 toxicities, both drugs were stopped permanently and for grade 3 toxicity (except for skin toxicity), sorafenib was delayed until less than or equal to grade 2 and then restarted at a dose decreased by one level. In case of grade 2 and 3 skin toxicity, paclitaxel was not interrupted while sorafenib was interrupted till resolution to grade 1 or less. The subsequent dose level of the drug was administered depending on its first, second, or more appearances. For grade 2 neurological toxicity, paclitaxel was administered at $80 \%$ of the dose, and in case of grade 3 toxicity, the drug was interrupted until return to baseline then restarted with $60 \%$ of planned dose.

Treatment evaluation The following tests were evaluated before entry onto the study: medical history and physical examination; performance status; weight; and routine laboratory tests: echocardiography, chest radiography, and chest and abdominal CT scan. Bone scan and brain CT (or magnetic resonance imaging) were performed if clinically indicated. An endocrine workup was also performed including measurement of serum and urinary cortisol, serum 17-hydroxyprogesterone, DHEAS, as well as testosterone in women and estradiol in men. Physical examination and hematological profile were repeated every week before starting paclitaxel administration and CT scan and endocrine workup were planned to be repeated every 8 weeks for the first 4 months and then every 12 weeks. In case of documented disease progression, the subjects were excluded from the study. Disease response was assessed using the RECIST criteria (21), while toxicity was graded using the NCI criteria.

Progression-free survival (PFS) was measured from the beginning of the treatment until the criteria for disease progression were met or last follow-up in nonprogressing patients. Overall survival was defined as the time interval between the date of beginning of treatment and the date of death from any cause or the last known alive date.

Plasma samples were collected for determining mitotane concentration at baseline and 3 and 6 weeks after starting paclitaxel and sorafenib treatment. Plasma mitotane was measured in-house by an HPLC assay, as described previously (22).
Cell culture and chemical reagents NCI-H295R cell line was supplied by the American Type Culture Collection (Rockville, MD, USA). Cells were cultured in a 1:1 mixture of DMEM and Ham's F-12 nutrient mixture (DMEM/F12; Sigma) supplemented with 1\% L-glutamine (Sigma) and 2.5\% Nu-Serum (Becton Dickinson, San Jose, CA, USA) and enriched with $1 \%$ of ITS + Premix (Becton Dickinson) at $37{ }^{\circ} \mathrm{C}$ in $5 \% \mathrm{CO}_{2}$ and $95 \%$ humidity. Sorafenib tosylate was a gift from Bayer Schering Pharma and paclitaxel was purchased from Calbiochem (Darmstadt, Germany); both drugs were dissolved in 100\% DMSO (Sigma).

Treatment and cell viability assay H295R cells were seeded at a density of $15 \times 10^{3}$ cells/100 $\mu \mathrm{l}$ in 96-well flat-bottomed plates (Corning, New York, NY, USA) in culture medium plus $1 \%$ ITS + Premix and $2.5 \%$ $\mathrm{Nu}$-Serum. After $48 \mathrm{~h}$, the medium was changed, and the cells were then cultured in medium alone (untreated conditions) or in medium containing different concentrations of drugs for different times. Cells were treated for 24 and $48 \mathrm{~h}$ with sorafenib $(1,5,10,25,50 \mu \mathrm{M})$ alone and with paclitaxel alone $(5,10,50,100,500$, $1000 \mathrm{nM}$ ) respectively. After incubation time, $10 \mu \mathrm{l}$ Cell Proliferation Reagent WST-1 (Roche Applied Science) were added to each well and incubated for $1 \mathrm{~h}$ at $37^{\circ} \mathrm{C}$ in order to measure cell proliferation. The absorbance was determined using a microplate (ELISA) reader at a test wavelength of $450 \mathrm{~nm}$ and reference wavelength of $630 \mathrm{~nm}$. Three replicate wells were used to determine each data point. The basal conditions were normalized to $100 \%$ for each assay and cell viability was expressed as a percentage of the untreated control. The mean percentage of inhibition of proliferation was calculated with respect to the control for each sample. To evaluate the possible synergic effect of sorafenib and paclitaxel on cell viability, H295R cells were exposed to fixed concentrations of sorafenib $(10$ or $50 \mu \mathrm{M})$ plus paclitaxel $(5,10,50,100,500,1000 \mathrm{nM})$ for 24 and $48 \mathrm{~h}$ respectively. After incubation time, cell viability was assessed as described above and the mean percentage of inhibition of proliferation was calculated with respect to treatment with 10 or $50 \mu \mathrm{M}$ sorafenib for each sample.

\section{Statistical analysis}

The primary end point of the study was to estimate PFS at a fixed time point of 4 months. PFS rate was planned to be evaluated in all the patients registered in the study, according to the intent to treat principle. The size of this trial was determined using the optimal two-stage phase II study design by Simon (23). Accordingly, the sample size was assessed in order to refuse a PFS rate of $15 \%$ (p0) after 4 months and to provide a statistical power of $80 \%$ in assessing the activity of the regimen as a PFS rate after 4 months of $40 \%$. The upper limit for the first-stage drug rejection was one nonprogressing patient after 4 months out of the first consecutive seven 
patients. The upper limit of the second-stage rejection was six nonprogressing patients after 4 months out of 25 consecutively enrolled patients. All survival functions were computed using the Kaplan-Meier method.

In vitro changes of proliferative activity induced by sorafenib and paclitaxel on ACC cells were analyzed by paired $t$-test. Data were expressed as mean \pm s.D. Statistical analysis was performed using SPSS for Windows Software (version 17; SPSS Inc., Casalecchio di Reno, Italy). Levels of statistical significance were set at $P$ value $<0.05$.

\section{Results}

This open-label, single-arm, multicenter phase II study was planned to be conducted in Italy, at the Medical Oncology Unit of the Azienda Ospedaliero Universitaria San Luigi di Orbassano, and in France, at the Service de Médecine Nucléaire et de Cancérologie Endocrinienne, Institut Gustave Roussy, Villejuif Cedex.

The study was open for enrollment in Italy in November 2008 and was closed to accrual in February 2009 due to inefficacy. According to the stopping rules adopted in this trial, the enrollment was interrupted when the first seven patients had been evaluated and disease progression observed in all of them at first radiological evaluation. At that time, ten patients had been enrolled.

\section{Patients and tumor characteristics}

Table 1 shows the patient characteristics of this series. Eight patients have been previously treated with surgery, five of them have been admitted to more than one surgical approach (median 3 (range 2-5)). Post-surgery radiation therapy was given to two patients. Two patients had metastatic disease at diagnosis and were treated with chemotherapy only. All patients had previously received mitotane therapy, five of them for advanced disease only, while in five patients mitotane was administered either in an adjuvant setting or for advanced disease. All patients did receive at least one chemotherapy line administered in association with mitotane. First-line chemotherapy consisted of cisplatin plus etoposide, plus/minus adriamycin. Four patients also received a second-line therapy consisting of streptozotocin plus mitotane (one patient), gemcitabine plus capecitabine plus mitotane (two patients), and carboplatin plus mitotane (one patient). Serum mitotane levels at baseline were $<1 \mathrm{mg} / \mathrm{ml}$ in three patients, between 3 and $4.5 \mathrm{ng} / \mathrm{ml}$ in four patients, around $7.5 \mathrm{ng} / \mathrm{ml}$ in two patients, while in the remaining patient, it was $18.7 \mathrm{ng} / \mathrm{ml}$.

\section{Treatment received}

Eight patients had received 8 weeks of treatment, one patient interrupted the treatment after 5 weeks due to deterioration of his performance status, and one patient interrupted the treatment after 2 weeks due to an acute abdomen. This adverse event resolved completely within 15 days, but then the patient refused further treatment. Both sorafenib and paclitaxel were interrupted in one patient for 1 week due to lung infection.

\section{Toxicity}

The treatment was well tolerated and the worst toxicities encountered are summarized in Table 2. None of them were dose limiting. The most relevant toxicity was fatigue that was observed in eight patients, being grade 2 or 3 in four patients. Three patients had severe hypophosphatemia (grade 3), accompanied by hypocalcemia in one of them (grade 2). Hematological and hepatic toxicities were mild. No patients had renal or skin toxicity.

Table 1 Patient characteristics.

\begin{tabular}{|c|c|}
\hline Characteristics & Value \\
\hline Total number & 10 \\
\hline Males & 4 \\
\hline Females & 6 \\
\hline Age (median (range); years) & $46(30-69)$ \\
\hline \multicolumn{2}{|l|}{ Performance status (ECOG) } \\
\hline 0 & 2 \\
\hline 1 & 8 \\
\hline Mitosis count ${ }^{\mathrm{a}}$ (median (range)) & $22(10-40)$ \\
\hline \multicolumn{2}{|l|}{ Stage at diagnosis } \\
\hline Locoregional & 8 \\
\hline Metastatic & 2 \\
\hline \multicolumn{2}{|l|}{ Secretory status at the study entry } \\
\hline Cortisol & 4 \\
\hline Cortisol + androgens & 2 \\
\hline Estradiol & 1 \\
\hline \multicolumn{2}{|l|}{ Primary treatment at diagnosis } \\
\hline Surgery & 8 \\
\hline Chemotherapy plus mitotane & 2 \\
\hline \multicolumn{2}{|l|}{ Number of surgical operations } \\
\hline 1 & 5 \\
\hline 2 & 2 \\
\hline$\geq 3$ & 3 \\
\hline Adjuvant mitotane & 5 \\
\hline \multicolumn{2}{|c|}{ Chemotherapy for advanced disease } \\
\hline \multicolumn{2}{|c|}{ First line } \\
\hline EDP + mitotane & 7 \\
\hline CDDP + VP16 & 2 \\
\hline CDDP + VP16 + mitotane & 1 \\
\hline \multicolumn{2}{|l|}{ Second line } \\
\hline Streptozotocin + mitotane & 1 \\
\hline Gemcitabine + capecitabine & 2 \\
\hline Carboplatin & 1 \\
\hline Radiation therapy & 2 \\
\hline \multicolumn{2}{|l|}{ Sites of disease } \\
\hline Lung & 8 \\
\hline Liver & 5 \\
\hline Lymph nodes & 4 \\
\hline Abdomen & 7 \\
\hline Bone & 1 \\
\hline \multicolumn{2}{|l|}{ Number of sites involved } \\
\hline 2 & 6 \\
\hline 3 & 4 \\
\hline
\end{tabular}

${ }^{\text {aper }} 50$ high power field. 
Table 2 Toxicity according to the $\mathrm{NCl}$ criteria. For each patient, the worst instance of toxicity was recorded.

\begin{tabular}{|c|c|c|c|c|c|}
\hline & \multicolumn{5}{|c|}{ Grades } \\
\hline & 0 & 1 & 2 & 3 & 4 \\
\hline Leukocytes (total WBC) & 9 & 1 & & & \\
\hline Platelets & 10 & & & & \\
\hline Hemoglobin & 8 & & 2 & & \\
\hline Creatinine & 10 & & & & \\
\hline Nausea/vomiting & 9 & 1 & & & \\
\hline Diarrhea & 9 & 1 & & & \\
\hline Stomatitis & 8 & 2 & & & \\
\hline Gamma-GT & 8 & & & 2 & \\
\hline AST/ALT & 9 & & 1 & & \\
\hline Bilirubin & 10 & & & & \\
\hline Alkaline phosphatase & 9 & 1 & & & \\
\hline Cardiovascular & 10 & & & & \\
\hline Neurology & 9 & 1 & & & \\
\hline Fever & 8 & 1 & 1 & & \\
\hline Alopecia & 10 & & & & \\
\hline Myalgia & 10 & & & & \\
\hline Skin toxicity & 10 & & & & \\
\hline Asthenia & 2 & 4 & 1 & 3 & \\
\hline Hypophosphatemia & 7 & & & 3 & \\
\hline Hypocalcemia & 9 & & 1 & & \\
\hline
\end{tabular}

\section{Treatment activity}

Disease response was assessable in nine patients; the patient who interrupted the treatment after 2 weeks was not evaluable for response. All evaluable patients showed a disease progression on target lesions at the first evaluation performed after 8 weeks. As shown in the waterfall diagram depicted in Fig. 1, in this short period of time, the tumor increase was dramatic: median $60 \%$ (range 26-152\%). In two patients, the progressive disease on target lesions was associated with the appearance of new lesions: mediastinal lymph nodes in one patient and lung metastases in one patient. Tumor progression was associated with a deterioration of performance status in eight patients. At the last followup, in December 2010, all but one patient was deceased.

\section{Cytotoxic activity of sorafenib and paclitaxel in vitro}

Sorafenib impaired cell viability of H295R cells either at $24 \mathrm{~h}$ (data not shown) or $48 \mathrm{~h}$ (Fig. 2a). A time course of sorafenib effects on cell growth and survival showed dose-response and time-response relationships. Conversely, treatment with paclitaxel alone showed a slight effect on cell viability (Fig. 2b). Moreover, H295R cells were treated with two fixed concentrations of sorafenib (10 and $50 \mu \mathrm{M})$ and a wide range of paclitaxel concentration (Fig. 2c and d). The overall effect of two drugs was similar to treatment with sorafenib alone at the same times and concentrations; indeed, no significant changes in viability ratio were detected with the combination of drugs with respect to sorafenib alone. A 24-h preincubation with paclitaxel, before treatment with sorafenib, showed the same response of concurrent administration (data not shown).

\section{Discussion}

ACC is a highly vascularized disease (24) and metronomic chemotherapy is thought to exert its anticancer activity mainly by inhibiting tumor angiogenesis. Moreover, the antiangiogenic activity of metronomic chemotherapy can be theoretically increased by the concomitant administration of an antiangiogenetic drug (4). Therefore, there is a strong rationale for testing the combination of metronomic chemotherapy plus antiangiogenetic drugs in the management of ACC patients.

Despite this sound biological basis, the combination of weekly paclitaxel and sorafenib was totally ineffective in ACC patients progressing after one or two chemotherapy lines. A clear progressive disease was observed in nine consecutive patients at the first restaging done after 2 months, and this led to interruption of the clinical trial.

It is noteworthy that in many patients, the tumor progression was dramatic and the increase in tumor lesions was greater and faster than that in the months before the trial. These data suggest that this combination therapy may have paradoxically favored tumor progression.

This treatment was well tolerated, although safety data are limited due to the short study duration.

In this population of heavily pretreated patients, some of them had grades 2-3 asthenia, anemia, and hypophosphatemia.

In a multicenter phase II trial previously done in Germany, ten patients who were heavily pretreated with three or more therapeutic lines received a combination of bevacizumab and capecitabine administered on a metronomic schedule (25). Similar to our data, all patients showed a disease progression (25).

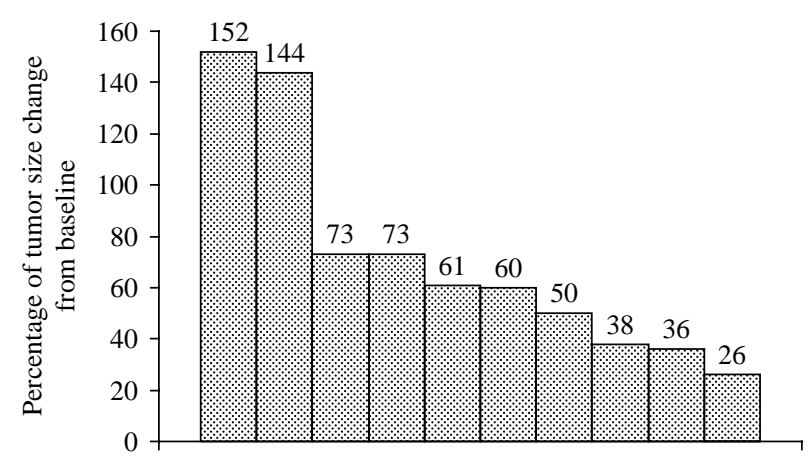

Figure 1 Waterfall diagram of percentage change in tumor size at first disease assessment. 

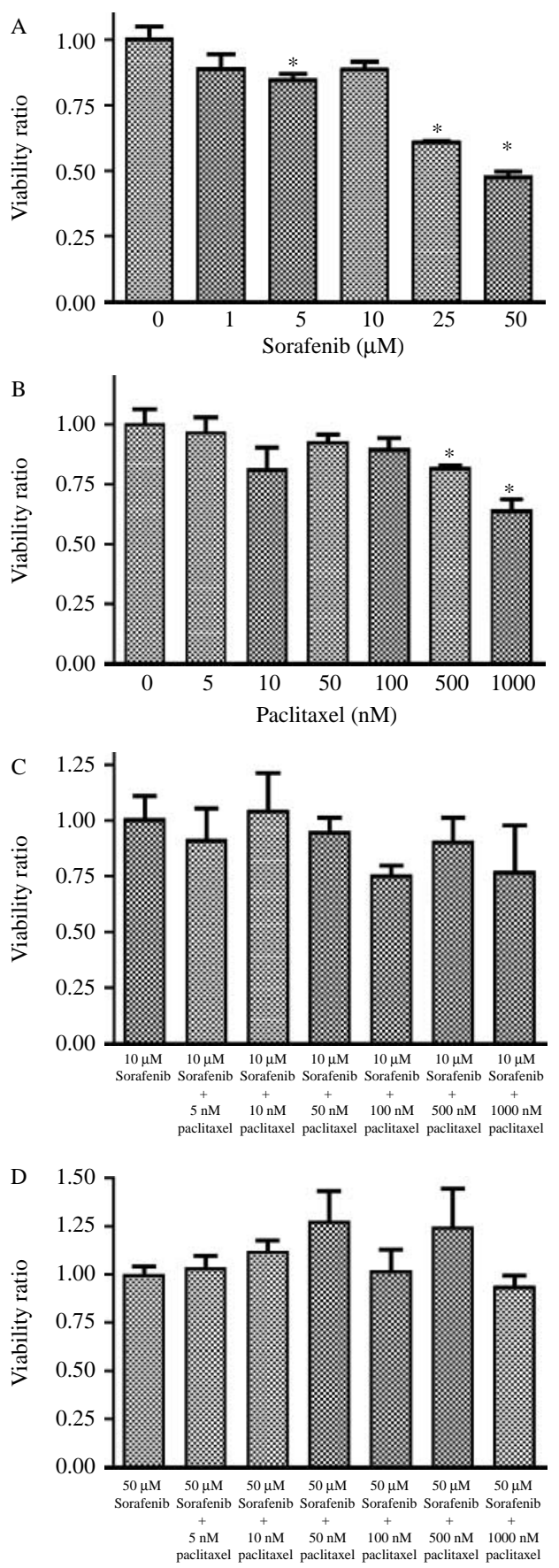

Figure 2 Cytotoxic effects of sorafenib, paclitaxel, and the combination of both drugs. The antiproliferative effect of sorafenib and paclitaxel alone and the combination of both drugs was assessed in vitro on H295R adrenocortical carcinoma cells. (A) $\mathrm{H} 295 \mathrm{R}$ cells were incubated for $48 \mathrm{~h}$ with different concentrations of sorafenib $(1,5,10,25$, and $50 \mu \mathrm{M})$ and (B) paclitaxel $(5,10,50$, $100,500,1000 \mathrm{nM}$ ). In addition, H295R cells were incubated for $48 \mathrm{~h}$ $(\mathrm{C}$ and $\mathrm{D})$ with two fixed concentrations of sorafenib $(10$ and $50 \mu \mathrm{M})$ and increasing concentrations of paclitaxel. Cell viability was determined by the WST-1 method and expressed as ratio between treated cells and untreated controls (basal); data are expressed as mean \pm s.D., $n=3$. Significance vs untreated cells, ${ }^{*} P<0.05$.
However, our in vitro study showed that ACC cells are sensitive to sorafenib administration. Paclitaxel, conversely, failed to be active against H295R ACC cells at variance with previous results (10). Paclitaxel, however, did not impair the antiproliferative effect of sorafenib, so the combination maintained an activity against ACC cells in vitro.

How can we explain our disappointing results? Of course, the great tumor burden and the multiple previous treatment lines could have been contributory. It should be noted, however, that both metronomic chemotherapy and sorafenib target angiogenesis and the combination of the two treatments may have induced tumor hypoxia in the cellular microenvironment leading to accelerated tumor growth $(26,27)$. It is known that tumor hypoxia is associated with a more aggressive phenotype in cancers. Tumor hypoxia occurs by stimulation of the hypoxia-inducible factor 1 transcription factor, which in turn stimulates a number of genes involved in either angiogenesis or tumor cell motility and invasion (28).

The finding of a U-shaped dose-response curve of antiangiogenic drugs activity provides support to this hypothesis (26).

A better understanding of how antiangiogenic drugs modify biology of ACC is therefore mandatory before further testing these drugs in ACC patients.

Our study, however, cannot exclude that sorafenib could be active if combined with other chemotherapeutic agents with known efficacy in ACC such as cisplatin (4).

We have recently shown that the combination of gemcitabine and metronomic capecitabine was active in heavily pretreated ACC patients (29). These findings suggest that a metronomic approach deserves interest despite the negative results of the present trial.

It should be noted, however, that mitotane was not withdrawn in the gemcitabine and capecitabine trial as in the present one, and mitotane serum levels within the therapeutic range $(14-20 \mathrm{ng} / \mathrm{ml})$ were associated with a prolonged PFS (29). These data suggest that mitotane may potentiate chemotherapy activity even in refractory disease. In the present study, all patients but one had mitotane levels consistently below the therapeutic range. On the other hand, it has been shown that sorafenib metabolism is altered by drugs able to increase hepatic p450 enzymes such as mitotane (30).

In conclusion, sorafenib plus weekly paclitaxel is a well-tolerated but inactive treatment in patients with advanced ACC and should not be recommended.

\section{Declaration of interest}

The authors declare that there is no conflict of interest that could be perceived as prejudicing the impartiality of the research reported. 


\section{Funding}

The study was supported in part by Bayer Healthcare, Regione Piemonte, Progetto Ricerca Finalizzata 2008 Number, Italian Association for Cancer Research (AIRC, Milan, grant no. IG/10795/2010), and Italian Ministry of the University and Scientific and Technological Research (PRIN, grant $n^{\circ} 20085$ P5S49).

\section{References}

1 Crucitti F, Bellantone R, Ferrante A, Boscherini M \& Crucitti P. The Italian Registry for Adrenal Cortical Carcinoma: analysis of a multiinstitutional series of 129 patients. The ACC Italian Registry Study Group. Surgery 1996119 161-170. (doi:10.1016/S00396060(96)80164-4)

2 Allolio B \& Fassnacht M. Clinical review: adrenocortical carcinoma: clinical update. Journal of Clinical Endocrinology and Metabolism 200691 2027-2037. (doi:10.1210/jc.2005-2639)

3 Assié G, Antoni G, Tissier F, Caillou B, Abiven G, Gicquel C, Leboulleux S, Travagli JP, Dromain C, Bertagna X, Bertherat J, Schlumberger M \& Baudin E. Prognostic parameters of metastatic adrenocortical carcinoma. Journal of Clinical Endocrinology and Metabolism 200792 148-154. (doi:10.1210/jc.2006-0706)

4 Berruti A, Ferrero A, Sperone P, Daffara F, Reimondo G, Papotti M, Dogliotti L, Angeli A \& Terzolo M. Emerging drugs for adrenocortical carcinoma. Expert Opinion on Emerging Drugs 200813 497-509. (doi:10.1517/14728214.13.3.497)

5 Kerbel RS \& Kamen BA. The anti-angiogenic basis of metronomic chemotherapy. Nature Reviews. Cancer 20044 423-436. (doi:10. 1038/nrc1369)

6 Bocci G, Nicolaou KC \& Kerbel RS. Protracted low-dose effects on human endothelial cell prolipheration and survival in vitro reveal a selective antiangiogenetic window for various chemotherapeutic drugs. Cancer Research 200262 638-643. (doi:10.1007/978-3540-78281-0_10)

7 Emmenegger U, Man S, Shaked Y, Francia G, Wong JW, Hicklin DJ \& Kerbel RS. A comparative analysis of low dose metronomic cyclophosphamide reveals absent or low-grade toxicity on tissues highly sensitive to the toxic effects of maximum tolerated dose regimens. Cancer Research 200464 3994-4000. (doi:10.1158/ 0008-5472.CAN-04-0580)

8 Emmenegger U, Francia G, Shaked Y \& Kerbel RS. Metronomic chemotherapy: principles and lessons learned from applications in the treatment of metastatic prostate cancer. Recent Results in Cancer Research 2010180 165-183.

9 Wiseman LR \& Spencer CM. Paclitaxel: an update of its use in the treatment of metastatic breast cancer and ovarian and other gynaecological cancers. Drugs \& Aging 199812 305-334. (doi:10.2165/00002512-199812040-00005)

10 Fallo F, Pilon C, Barzon L, Pistorello M, Pagotto U, Altavilla G, Boscaro $M$ \& Sonino N. Paclitaxel is an effective antiproliferative agent on the human NCI-H295 adrenocortical carcinoma cell line. Chemotherapy 199844 129-134. (doi:10.1159/ $000007104)$

11 Green MC, Buzdar AU \& Smith T. Weekly paclitaxel improves pathologic complete remission in operable breast cancer when compared with paclitaxel once every 3 weeks. Journal of Clinical Oncology 200523 5983-5992. (doi:10.1200/JCO.2005.06.232)

12 Seidman AD, Berry D, Cirrincione C, Harris L, Muss H, Marcom P, Gipson G, Burstein H, Lake D, Shapiro CL, Ungaro P, Norton L, Winer E \& Hudis C. Randomized phase III trial of weekly compared with every-3-weeks paclitaxel for metastatic breast cancer, with trastuzumab for all HER-2 overexpressors and random assignment to trastuzumab or not in HER-2 nonoverexpressors: final results of cancer and leukemia group B protocol 9840. Journal of Clinical Oncology 200826 1642-1649. (doi:10.1200/JCO.2007.11.6699)

13 Cazejust J, De Baère T, Auperin A, Deschamps F, Hechelhammer L, Abdel-Rehim M, Schlumberger M, Leboulleux S \& Baudin E. Transcatheter arterial chemoembolization for liver metastases in patients with adrenocortical carcinoma. Journal of Vascular and Interventional Radiology 201021 1527-1532. (doi:10.1016/j.jvir. 2010.05.020)

14 Bernini GP, Moretti A, Bonadio AG, Menicagli M, Viacava P, Naccarato AG, Iacconi P, Miccoli P \& Salvetti A. Angiogenesis in human normal and pathologic adrenal cortex. Journal of Clinical Endocrinology and Metabolism 2002287 4961-4965. (doi:10.1210/jc.2001-011799)

15 Wilhelm SM, Carter C, Tang L, Wilkie D, Mcnabola A, Rong H, Chen C, Zhang X, Vincent P, McHugh M, Cao Y, Shujath J, Gawlak S, Eveleigh D, Rowley B, Liu L, Adnane L, Lynch M, Auclair D, Taylor I, Gedrich R, Voznesensky A, Riedl B, Post LE \& Bollag G. BAY43-9006 exhibits broad spectrum oral antitumor activity and targets the RAF/MEK/ERK pathway and receptor tyrosine kinases involved in tumor progression and angiogenesis. Cancer Research 200464 7099-7109. (doi:10.1158/0008-5472. CAN-04-1443)

16 Hasskarl J. Sorafenib. Cancer Research 2010184 61-70.

17 Klement G, Baruchel S, Rak J, Man S, Clark K, Hicklin DJ, Bohlen P $\&$ Kerbel RS. Continuous low dose therapy with vinblastine and VEGF receptor-2 antibody induces sustained tumor regression without overt toxicity. Journal of Clinical Investigation $2000 \mathbf{1 0 5}$ R15-R24. (doi:10.1172/JCI8829)

18 Browder T, Butterfield CE, Kraling BM, Marshall B, O’Reilly MS \& Folkman J. Antiangiogenic scheduling of chemotherapy improves efficacy against experimental drug-resistant cancer. Cancer Research $2000601878-1886$.

19 Garcia AA, Hirte H, Fleming G, Yang D, Tsao-Wei DD, Roman L, Groshen S, Swenson S, Markland F, Gandara D, Scudder S, Morgan R, Chen H, Lenz HJ \& Oza AM. Phase II clinical trial of bevacizumab and low-dose metronomic oral cyclophosphamide in recurrent ovarian cancer: a trial of the California, Chicago, and Princess Margaret Hospital phase II consortia. Journal of Clinical Oncology 200826 76-82. (doi:10.1200/JCO.2007.12.1939)

20 Dellapasqua S, Bertolini F, Bagnardi V, Campagnoli E, Scarano E, Torrisi R, Shaked Y, Mancuso P, Goldhirsch A \& Rocca A. Metronomic cyclophosphamide and capecitabine combined with bevacizumab in advanced breast cancer. Journal of Clinical Oncology 200826 4899-4905. (doi:10.1200/JCO.2008.17. 4789)

21 Therasse P, Arbuck SG, Eisenhauer EA, Wanders J, Kaplan RS, Rubinstein L, Verweij J, Van Glabbeke M, van Oosterom AT \& Christian MC. New guidelines to evaluate the response to treatment in solid tumors. European Organization for Research and Treatment of Cancer, National Cancer Institute of the United States, National Cancer Institute of Canada. Journal of the National Cancer Institute 200092 205-216. (doi:10.1093/jnci/92.3.205)

22 De Francia S, Pirro E, Zappia F, De Martino F, Sprio AE, Daffara F, Terzolo M, Berruti A, Di Carlo F \& Ghezzo F. A new simple HPLC method for measuring mitotane and its two principal metabolites. Tests in animals and mitotane-treated patients. Journal of Chromatography. B, Analytical Technologies in the Biomedical and Life Sciences 2006837 69-75. (doi:10.1016/j.jchromb.2006.04. 005)

23 Simon R. Optimal two-stage designs for phase II clinical trials. Controlled Clinical Trials 198910 1-10. (doi:10.1016/01972456(89)90015-9)

24 de Fraipont F, El Atifi M, Gicquel C, Bertagna X, Chambaz EM \& Feige JJ. Expression of the angiogenesis markers vascular endothelial growth factor- $A$, thrombospondin-1, and plateletderived endothelial cell growth factor in human sporadic adrenocortical tumors: correlation with genotypic alterations. Journal of Clinical Endocrinology and Metabolism 200085 47344741. (doi:10.1210/jc.85.12.4734)

25 Wortmann S, Quinkler M, Ritter C, Kroiss M, Johanssen S, Hahner S, Allolio B \& Fassnacht M. Bevacizumab plus capecitabine as a salvage therapy in advanced adrenocortical carcinoma. European Journal of Endocrinology 2010162 349-356. (doi:10. 1530/EJE-09-0804)

26 Paez-Ribes M, Allen E, Hudock J, Takeda T, Okuyama H, Viñals F, Inoue M, Bergers G, Hanahan D \& Casanovas O. Antiangiogenic 
therapy elicits malignant progression of tumors to increased local invasion and distant metastasis. Cancer Cell 200915 220-231. (doi:10.1016/j.ccr.2009.01.027)

27 Steeg PS. Angiogenesis inhibitors: motivators of metastasis? Nature Medicine 20039 822-823. (doi:10.1038/nm0703-822)

28 Peinado $\mathrm{H} \&$ Cano A. A hypoxic twist in metastasis. Nature Cell Biology 200810 253-254. (doi:10.1038/ncb0308-253)

29 Sperone P, Ferrero A, Daffara F, Priola A, Zaggia B, Volante M, Santini D, Vincenzi B, Badalamenti G \& Intrivici C. Gemcitabine plus metronomic 5-fluorouracil or capecitabine as a second-/third-line chemotherapy in advanced adrenocortical carcinoma: a multicenter phase II study. Endocrine-Related Cancer 201017 445-453. (doi:10.1677/ERC-09-0281)

30 Hahner S \& Fassnacht M. Mitotane for adrenocortical carcinoma treatment. Current Opinion in Investigational Drugs 20056 386-394.

Received 25 October 2011

Revised version received 7 December 2011

Accepted 20 December 2011 